\title{
A SEARCH FOR SQUARK AND GLUINO PRODUCTION AT THE CERN $\bar{p}$ P COLLIDER
}

\section{The UA2 Collaboration}

Bern - Cambridge - CERN - Heidelberg - Milano -

Orsay (LAL) - Pavia - Perugia - Pisa - Saclay (CEN)

J.Alitti $^{10}$, R.Ansari ${ }^{6}$, R.E.Ansorge ${ }^{2}$, P.Bagnaia ${ }^{3, a}$, P.Bareyre ${ }^{10}$, G. Blaylock ${ }^{3}$, P.Bonamy ${ }^{10}$, M.Bonesini ${ }^{5,3}$, K.Borer ${ }^{1}$, D.Buskulic ${ }^{6}$, G.Carboni ${ }^{9}$, D.Cavalli ${ }^{5}$, V.Cavasinni ${ }^{9}$, P.Cenci ${ }^{8}$, J.C.Chollet ${ }^{6}$, C.Conta ${ }^{7}$, G.Costa ${ }^{5}$, F.Costantini ${ }^{9,3}$, A.Dell'Acqua ${ }^{7}$, B.De Lotto ${ }^{7, b}$, T.Del Prete ${ }^{9}$, R.S.DeWolf ${ }^{2}$, L.Di Lella ${ }^{3}$, G.F.Egan ${ }^{3, c}$, K.F.Einsweiler $^{3}$, L.Fayard ${ }^{6}$, A.Federspiel ${ }^{1}$, R.Ferrari ${ }^{7}$, M.Fraternali ${ }^{7, d}$, D.Froidevaux ${ }^{6}$, G.Fumagalli $i^{3}, 7$, J.M.Gaillard ${ }^{6}$, R.Geiges ${ }^{4}$, F.Gianotti ${ }^{5}$, O.Gildemeister ${ }^{3}$, C.Gössling ${ }^{3, e}$, V.G.Goggi ${ }^{7}, 3$, S.Grünendahl ${ }^{4}$, J.R.Hansen ${ }^{3}$, K.Hara ${ }^{1, f}$, S.Hellman $^{3}$, E.Hugentobler ${ }^{1}$, K.Hultqvist ${ }^{3}$, E.Iacopini ${ }^{9}$, , J.Incandela ${ }^{3}$, K.Jakobs ${ }^{3}$, P.Jenni ${ }^{3}$, E.E.Kluge ${ }^{4}$, T.Koch ${ }^{4}$, N.Kurz ${ }^{4}$, S. Lami ${ }^{9}$, P.Lariccia ${ }^{8}$, M.Lefebvre ${ }^{2,3}$, L.Linssen ${ }^{3}$, M.Livan ${ }^{7, h}$, P.Lubrano ${ }^{3}$,

C.Magneville ${ }^{10}$, L.Mandelli ${ }^{5}$, L.Mapelli ${ }^{3}$, M.Mazzanti ${ }^{5}$, K.Meier ${ }^{3}$, B.Merkel ${ }^{6}$, J.P.Meyer ${ }^{10}$, M.Moniez ${ }^{6}$, R.Moning ${ }^{1}$, M.Morganti, ${ }^{9}$, , L.Müller ${ }^{1}$, D.J.Munday ${ }^{2}$, C.Onions ${ }^{3}$, T.Pal ${ }^{3,1}$, M.A.Parker ${ }^{3}$, G.Parrour ${ }^{6}$, F.Pastore ${ }^{7}$, E.Pennacchio ${ }^{7}$,

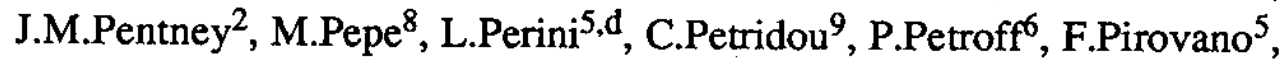
H.Plothow-Besch ${ }^{4}$, G.Polesello ${ }^{5,3}$, A.Poppleton ${ }^{3}$, L.Rasmussen ${ }^{3}$, J.P.Repellin ${ }^{6}$, A.Rimoldi ${ }^{7}$, P.Scampoli ${ }^{8}$, J.Schacher ${ }^{1}$, S.L.Singh ${ }^{2}$, S.Stapnes ${ }^{3}$,A.V.Stirling ${ }^{10}$, S.N.Tovey ${ }^{3, c}$, M.Valdata-Nappi ${ }^{9, j}$, V.Vercesi ${ }^{3,7}$, G.Volpini ${ }^{5}$, A.R.Weidberg ${ }^{3}$, P.S.Wells ${ }^{2}$, T.O.White ${ }^{2}$, D.R.Wood ${ }^{3}$, S.A.Wotton ${ }^{2}$, H.Zaccone ${ }^{10}$ 


\begin{abstract}
A search has been made with the upgraded UA2 experiment at the CERN $\overline{\mathrm{p}} \mathrm{p}$ Collider at $V_{\mathrm{s}}=630 \mathrm{GeV}$ for multi-jet events with large transverse momentum imbalance in a data sample corresponding to an integrated luminosity of $7.4 \mathrm{pb}^{-1}$. Under the assumption that the photino is the lightest stable supersymmetric (SUSY) particle, the data have been used to deduce lower limits in a minimal SUSY model for the squark (mq) and the gluino $\left(m_{\tilde{g}}\right)$ masses. The $90 \%$ C.L. bounds are $\mathrm{m}_{\tilde{q}}>74 \mathrm{GeV}$ independent of $\mathrm{m}_{\tilde{\mathrm{g}}}$, $\mathrm{m} \tilde{g}>79 \mathrm{GeV}$ independent of $\mathrm{m} \tilde{q}$, and $m>106 \mathrm{GeV}$ for $\mathrm{m}_{\tilde{q}}=\mathrm{m}_{\mathfrak{g}}=\mathrm{m}$.
\end{abstract}

1 Laboratorium für Hochenergiephysik, Universităt Bern, Sidlerstraße 5, 3012 Bern, Switzerland

2 Cavendish Laboratory, University of Cambridge, Cambridge, CB3 OHE, UK

3 CERN, 1211 Geneva 23, Switzerland

4 Institut für Hochenergiephysik der Universităt Heidelberg, Schröderstraße 90 , 6900 Heidelberg, FRG

5 Dipartimento di Fisica dell'Università di Milano and Sezione INFN Milano, 20133 Milano, Italy

6 Laboratoire de l'Accélérateur Linéaire, Université de Paris-Sud, 91405 Orsay, France

7 Dipartimento di Fisica Nucleare e Teorica, Università di Pavia and INFN, Sezione di Pavia, Via Bassi 6, 27100 Pavia, Italy

8 Dipartimento di Fisica dell'Università di Perugia and INFN, Sezione di Perugia, via Pascoli, 06100 Perugia, Italy

9 Dipartimento di Fisica dell'Università di Pisa and INFN, Sezione di Pisa, Via Livornese, S.Piero a Grado, 56100 Pisa, Italy

10 Centre d'Etudes Nucléaires de Saclay, 91191 Gif-sur-Yvette Cedex, France

a) Now at Dipartimento di Fisica, Università di Roma, Italy

b) Now at Dipartimento di Fisica, Università di Udine, Italy

c) Visitor from the University of Melbourne, Parkville, Australia 3052

d) Now at Dipartimento di Fisica, Università di Palermo, Italy

e) Now at Institut für Physik, Universităt Dortmund, FRG

f) Now at University of Tsukuba, Tsukuba, Ibaraki 305, Japan

g) Also at Scuola Normale Superiore, Pisa, Italy

h) Now at Dipartimento di Fisica, Università di Cagliari, Italy

i) Now at Dipartimento di Fisica e INFN di Bologna, Universita Bolognia, Italy

j) Now at Dipartimento di Fisica dellUniversità della Calabria, Cosenza, Italy 


\section{INTRODUCTION}

Hadron colliders provide the possibility to search for hypothetical new particles beyond the Standard Model over a large mass range. In particular, abundant QCD production of squarks ( $(\widetilde{\mathrm{q}})$ and gluinos ( $(\widetilde{\mathrm{g}})$ is predicted in supersymmetric (SUSY) models [1], the dominant sources of SUSY particles being $\widetilde{\mathrm{q}} \overline{\mathrm{q}}$, $\widetilde{\mathrm{q}} \widetilde{\mathrm{g}}$ and $\widetilde{\mathrm{gg}}$ pair production. In most models the photino $(\widetilde{\gamma})$ is expected to be the lightest SUSY particle (LSP), and hence stable if R-parity conservation is assumed. In these models one expects that the $\widetilde{\mathrm{g}}$ decays mainly into $\mathrm{q} \overline{\mathrm{q}} \tilde{\gamma}$ and the $\widetilde{\mathrm{q}}$ dominantly into $\mathrm{q} \widetilde{\mathrm{g}}$ (if $\mathrm{m}_{\tilde{\mathrm{q}}}>\mathrm{m}_{\tilde{\mathrm{g}}}$ ) or into $\mathrm{q} \tilde{\gamma}$ (if $\mathrm{m}_{\tilde{\mathrm{q}}}<\mathrm{m} \widetilde{\mathrm{g}}$ ), at least for the mass range accessible at the CERN $\bar{p}$ collider [2]. In this case, the hadronic production of $\widetilde{\mathrm{q}}$ and $\tilde{\mathrm{g}}$ results in final states containing two to six jets and missing transverse momentum $\left(p_{\mathrm{T}}\right)$ due to the undetected photinos.

A search has been made with the upgraded UA2 experiment at the improved CERN $\overline{\mathrm{p} p}$ Collider at $\sqrt{\mathrm{s}}_{\mathrm{s}}=630 \mathrm{GeV}$ [3] for multi-jet events with large $p_{\mathrm{T}}$. A large data sample has been collected in the 1988 and 1989 running periods corresponding to an integrated luminosity of $7.4 \mathrm{pb}^{-1}$,which is much larger than that used in previous $\widetilde{\mathrm{q}}$ and $\widetilde{\mathrm{g}}$ searches at the CERN $\bar{p} p$ Collider [4-6] and at FNAL Tevatron Collider [7] at $\sqrt{ } s=1.8 \mathrm{TeV}$. No event passes simple kinematical cuts for which a significant number of SUSY particles with masses above the present lower limits [5-7] would remain observable with the present data sample.

This Letter is organized as follows. In Sect. 2 the main aspects of the upgraded UA2 detector which are relevant for this analysis are described. An account of the data reduction and of the selection criteria is contained in Sect. 3. The SUSY model used to extract mass limits is described in Sect. 4, followed by a discussion of the systematic uncertainties and the resulting $\widetilde{\mathrm{q}}$ and $\widetilde{\mathrm{g}}$ mass limits in Sect. 5 .

SUSY particles without strong interaction can be searched for at the CERN $\bar{p} p$ Collider in the decays of Intermediate Vector Bosons (IVB). Such a search through $Z$ decays from data collected by UA2 during the same running periods is presented in [8].

\section{EXPERIMENTAL ASPECTS}

\subsection{The upgraded UA2 detector}

In order to exploit the improved CERN $\vec{p}$ Collider, the UA2 experiment has been considerably upgraded and rebuilt [9], with two main aims : full calorimeter coverage for good $\phi_{\mathrm{T}}$ measurements and enhanced electron identification, particularly at low transverse momenta $\left(\mathrm{p}_{\mathrm{T}}\right)$. The first aspect is crucial for $\widetilde{\mathrm{q}}$ and $\widetilde{\mathrm{g}}$ searches. Emphasis is therefore given 
in the following to a description of the new calorimetry of UA2 $[9,10]$. An improved electron signature has been achieved with a novel detector assembly combining in cylindrical layers tracking and electron identification detectors [9]. In addition to the $p_{\mathrm{T}}$ measurement this second aspect is also basic to the UA2 search for electroweakly interacting SUSY particles, and is therefore described in more detail in Ref. [8].

The increased collider luminosity also required a new sophisticated multi-level trigger and data acquisition system [11].

The calorimetry of the upgraded UA2 detector is shown in Fig. 1. The pseudorapidity $(\eta)$ coverage of the central calorimeter [12] of $-1<\eta<1$ has been extended with new electromagnetic (em) and hadronic end cap calorimeters to $-3<\eta<3$. The end cap calorimeters use the same technique as the central calorimeter $(\mathrm{Pb}$ and $\mathrm{Fe}$ absorber plates with scintillator and wave-length-shifter read-out), with similar cell granularity and sampling fractions. The two cells closest to the beam axis $(2.5<|\eta|<3.0$ and $2.2<|\eta|<2.5)$ cover $30^{\circ}$ in azimuth $(\phi)$. The other end cap cells have a constant segmentation of $\Delta \phi=15^{\circ}$, $\Delta \eta=0.2$. All the cells in $1.0<|\eta|<2.5$ have one em and one hadronic compartment, the cell closest to the beam has only a hadronic compartment. Special emphasis has been given to good hermeticity (minimal dead space) of the calorimetry in order to achieve excellent $p_{\mathrm{T}}$ measurements. To this purpose, additional hadronic calorimeter cells cover the interval $0.9<|\eta|<1.0$, overlapping with the edge cells of the central calorimeter (see Fig. 1), to measure particles escaping from the interface between the end caps and the central calorimeter. Also, in order to avoid an alignment of the dead space occupied by the wavelength-shifter read-out plates with the event vertex, each individual end cap module $\left(\Delta \phi=30^{\circ}\right)$ is tilted by an angle of $50 \mathrm{mrad}$ with respect to a plane perpendicular to the beam axis. The energy resolution $\sigma_{\mathrm{E}}$ for electromagnetic showers is typically $0.17 \mathrm{E}^{1 / 2}$ and for hadronic showers $0.68 \mathrm{E}^{1 / 2}$ for $\mathrm{E}$ below $25 \mathrm{GeV}$ and $0.30 \mathrm{E}^{3 / 4}$ above $\left(\mathrm{E}\right.$ in $\mathrm{GeV}$ ). The $p_{\mathrm{T}}$ resolution will be discussed in the next Section. Further details on the construction and the performance of the calorimeters can be found in Ref. [10].

Two planes of large area scintillation counters (VETO counters) cover the back sides of the end cap calorimeters. Their purpose is to signal beam-halo background particles accompanying the $p$ and $\bar{p}$ bunches. Particles which give an early signal with respect to the beam crossing time can easily be rejected in the analysis.

Also indicated in Fig. 1 are the locations of the time-of-flight (TOF) hodoscopes which cover a $\eta$-interval of $2.3<|\eta|<4.1$. The TOF arrays, consisting of 20 counters on each side, give a coincidence signal from both sides of the interaction region for about $98 \%$ of hard inelastic $\bar{p}$ interactions. For a single counter the particle time-of-flight from the interaction vertex to the TOF array is measured with an accuracy of about $0.3 \mathrm{~ns}$ (rms). This results in a vertex determination along the beam axis with a rms precision of $2.5 \mathrm{~cm}$. The 
good TOF resolution is exploited in the present analysis to suppress backgrounds from beam-halo or beam-beam interactions not coming from the interaction region.

\subsection{Missing $\mathbf{p}_{\mathrm{T}}$ resolution}

In a $\overline{\mathrm{p} p}$ interaction the missing transverse momentum of an event is defined as

$$
\vec{p}_{\mathrm{T}}=-\sum_{\mathrm{i}} \overrightarrow{\mathrm{P}}_{\mathrm{T}}^{\mathrm{i}}
$$

where $\overrightarrow{\mathrm{P}}_{\mathrm{T}}^{\mathrm{i}}$ are the transverse momentum vectors of the emitted particles. In an ideal detector, if all particles are detected, $\phi_{\mathrm{T}}=0$. If, however, one or more non-interacting particles are emitted, $\vec{b}_{\mathrm{T}}$ represents the vector sum of their transverse momenta. Experimentally relation (1) is approximated by

$$
\vec{\phi}_{\mathrm{T}}=-\Sigma_{\mathrm{j}} \mathrm{E}_{\mathrm{T}}^{\mathrm{j}} \overrightarrow{\mathrm{n}}_{\mathrm{j}}
$$

where $\vec{n}_{j}$ is a unit vector in the transverse plane pointing from the event vertex to the centre of cell $\mathrm{j}$ with transverse energy $\mathrm{E}_{\mathrm{T}}$. The sum extends over all calorimeter cells.

It is observed experimentally that for unbiased event samples of a given total transverse energy $\mathrm{E}_{\mathrm{T}}$, defined as the scalar sum of all cell $\mathrm{E}_{\mathrm{T}} \mathbf{j}$, any two orthogonal components $(\mathrm{x}, \mathrm{y})$ of $\vec{p}_{\mathrm{T}}$ have Gaussian distributions, centred at zero and with standard deviations $\sigma_{\mathrm{x}}=\sigma_{\mathrm{y}}=\sigma\left(\mathrm{E}_{\mathrm{T}}\right)$. Therefore the distribution of $p_{\mathrm{T}}^{2}$ can be described by

$$
\frac{\mathrm{dn}}{\mathrm{d}\left(\phi_{\mathrm{T}}\right)^{2}} \sim\left(1 / \Delta^{2}\right) \exp \left[-\left(\phi_{\mathrm{T}} / \Delta\right)^{2}\right]
$$

with $\Delta=\sqrt{ } 2 \cdot \sigma$. Figure 2 shows two $\mathrm{dn} / \mathrm{d}\left(\boldsymbol{p}_{\mathrm{T}}\right)^{2}$ distributions for two representative $\mathrm{E}_{\mathrm{T}}$ intervals. These data have been collected free of any azimuthal or $\eta$ trigger bias. The experimental distributions are consistent with an exponential decrease as a function of $p_{T}^{2}$. No significant non-Gaussian tails are present up to $p_{\mathrm{T}}$ values of about $4 \Delta$. Such tails could be caused by non-hermetic calorimeter coverage. The parameter $\Delta$ is a function of $\mathrm{E}_{\mathrm{T}}$ and is well parametrized by the form $\Delta=\alpha\left(\mathrm{E}_{\mathrm{T}}\right)^{\beta}$ with $\alpha=0.8$ and $\beta=0.4$. 


\subsection{Data taking}

The data used for this analysis were collected with a dedicated $p_{\mathrm{T}}$ trigger. The first level $p_{\mathrm{T}}$ trigger, based on a purpose - built fast electronics module [13], applied a threshold of $15 \mathrm{GeV}$ to the $p_{\mathrm{T}}$ calculated from the calorimeter information for $\eta \mid<2$. At the first level, a coincidence was required with a beam-beam interaction signal from the TOF counters in order to suppress backgrounds from beam-halo interactions. The efficiency of this requirement was measured to be $99.1 \pm 0.1 \%$ for unambiguous two-jet events. The second and third level triggers improved the event selection by using the full calorimeter coverage and refined calibration constants. The final selection was fully efficient for events with $p_{\mathrm{T}}>20 \mathrm{GeV}$. Typical trigger rates were $8 \mathrm{~Hz}$ at the first level and $0.2 \mathrm{~Hz}$ accepted at the third level for a $\bar{p}$ C Collider luminosity of $2 \cdot 10^{30} \mathrm{~cm}^{-2} \mathrm{~s}^{-1}$.

Data were accumulated in the 1988 and $1989 \overline{\mathrm{p} p}$ Collider runs at $V_{\mathrm{s}}=630 \mathrm{GeV}$ corresponding to an integrated luminosity of $7.4 \pm 0.5 \mathrm{pb}^{-1}$.

\section{DATA REDUCTION}

Jets are defined as energy clusters of adjacent calorimeter cells with energy larger than $400 \mathrm{MeV}$, the jet direction pointing from the event vertex to the energy weighted centre of the cluster. The jets are ordered according to decreasing transverse energy as $\mathrm{E}_{\mathrm{T}}^{1}>\mathrm{E}_{\mathrm{T}}^{2}>$ $\mathrm{E}_{\mathrm{T}}^{3}$... . A series of selection criteria is used to identify a possible signal of true multi-jet events with large $p_{\mathrm{T}}$. Four types of backgrounds must be rejected :

i) Beam-halo interactions in coincidence with an accepted TOF beam-beam trigger.

ii) Multi-jet events from $\bar{p}$ p interactions not occuring within the nominal vertex region due to beam particles in RF buckets adjacent to those of the colliding bunches. In this case a fake $p_{\mathrm{T}}$ can be created at the trigger level by evaluating the $\mathrm{E}_{\mathrm{T}}^{j}$ with respect to the centre of the detector.

iii) Genuine multi-jet events with mis-measured transverse energies, particularly for jets near the boundaries between the central and the end cap calorimeters.

iv) Multi-jet events from conventional processes with neutrino or muon emission.

The beam-halo background (i) can be removed completely with the following selection criteria. The events are required to have a total energy of less than $630 \mathrm{GeV}$ and the leading jet $\left(\mathrm{E}_{\mathrm{T}}^{1}\right)$ has to have at least $5 \%$ of its energy deposited in the em part of the calorimeter. In addition, no early VETO counter signals are allowed to be present in the 
event. The loss of real events due to these selections has been measured to be negligible $(<1 \%)$ from a sample of unambiguous two-jet events .

The TOF measurements are used to retain only events produced by $\bar{p} p$ reactions within the nominal collision region and to eliminate background (ii). The contamination from such events in the $p_{\mathrm{T}}$ data sample varies between 20 and $50 \%$ depending on the machine conditions. Events are rejected if they do not have a recognized TOF vertex, or if at least 5 out of the 20 TOF counters on either side have timing signals incompatible with the nominal beam crossing time. The efficiency of the TOF criteria has been measured with a large sample of two-jet events to be $97.5 \pm 0.5 \%$. The $\dot{p}_{\mathrm{T}}$ is then recomputed using the TOF vertex.

Kinematical criteria are applied at this stage to reduce backgrounds from source (iii) and to optimize a possible SUSY signal over the QCD multi-jet background. At least two jets are required to be present in the event with $\mathrm{E}_{\mathrm{T}}^{1}>25 \mathrm{GeV}$ and $\mathrm{E}_{\mathrm{T}}^{2}>15 \mathrm{GeV}$ within the central region $|\eta|<0.85$. Exclusive QCD two-jet events, defined as having $\mathrm{E}_{\mathrm{T}}^{3}<10 \mathrm{GeV}$, are rejected if they occur in their dominant configuration, namely with back-to-back jets in the transverse plane. Only those events are accepted for which the two-jet azimuthal separation satisfies $\Delta \phi\left(\mathrm{E}_{\mathrm{T}}^{1}, \mathrm{E}_{\mathrm{T}}^{2}\right)<160^{\circ}$. About 2100 events with $p_{\mathrm{T}}>20 \mathrm{GeV}$ survive all the selection criteria listed so far. Their $\phi_{\mathrm{T}}{ }^{2}$ distribution, shown in Fig. 3, can be characterized by an exponential decrease for $20<p_{\mathrm{T}} \leq 40 \mathrm{GeV}$ and by a tail extending to very large $p_{\mathrm{T}}$ values. This tail is expected to include the region of the Jacobian peak due to the neutrino from $W$ decays of background (iv).

The QCD multi-jet background with $\phi_{\mathrm{T}}$ from mis-measured jets can be further reduced by isolation requirements on the $\vec{p}_{\mathrm{T}}$ vector with respect to the jet directions in the transverse plane. The following two conditions are applied to retain events : the $p_{\mathrm{T}}$ is not back-to-back to the leading jet $\left[\Delta \phi\left(\mathrm{E}_{\mathrm{T}}^{1}, p_{\mathrm{T}}\right)<140^{\circ}\right]$ and it is not aligned in azimuth with any jet $\mathrm{E}_{\mathrm{T}}^{3}, \mathrm{E}_{\mathrm{T}}^{4} \ldots>10 \mathrm{GeV}\left[\Delta \phi\left(\mathrm{E}_{\mathrm{T}}^{\mathrm{k}}, \phi_{\mathrm{T}}\right)>20^{\circ}, \mathrm{k}=3,4 \ldots\right]$.

Finally there remains the physics background (iv) from IVB production in association with jets, followed by IVB decays with neutrino emission giving rise to real $p_{\mathrm{T}}$. Part of this background involves an electron in the final state. Therefore events containing identified electrons have been removed. The electrons have been defined by purely calorimetric criteria based on the lateral and longitudinal shower size. These criteria remove isolated electrons with an efficiency close to $100 \%$, as estimated from fully identified $\mathrm{W} \rightarrow \mathrm{ev}$ decays, whereas only $2.1 \pm 0.3 \%$ of two-jet events are lost.

The $p_{\mathrm{T}}^{2}$ distribution after the full data reduction is shown as the shaded histogramme in Fig. 3. The tail of very large $p_{\mathrm{T}}$ events has been identified by the last two groups of criteria as mis-measured QCD jet or Standard Model IVB events and removed completely from the initial sample. The remaining events are compatible with the QCD jet background 
expected from $p_{\mathrm{T}}$ resolution fluctuations as indicated by their exponential decrease as a function of $p_{T}^{2}$. This conclusion is also supported by an explicit preliminary evaluation of the Standard Model processes for IVB +1 or 2 jet production ( $\leq 6$ events), a topic outside the scope of the present Letter. No event survives the final selection $p_{\mathrm{T}}>40 \mathrm{GeV}$. Taking into account the efficiencies of the electron rejection and TOF based criteria, as well as the error on the integrated luminosity, this corresponds to an upper limit on the observed cross section of $0.35 \mathrm{pb}(90 \% \mathrm{CL})$ for the selected kinematical and topological configurations.

\section{MONTE CARLO SIMULATION}

\subsection{The SUSY model}

Large samples of $\widetilde{\mathrm{q}} \overline{\tilde{\mathrm{q}}}, \widetilde{\mathrm{qg}}$, and $\widetilde{\mathrm{gg}}$ events have been generated for various $\mathrm{m} \widetilde{\mathrm{q}}$ and $\mathrm{m} \widetilde{\mathrm{g}}$ values in order to evaluate the detector sensitivity to a possible SUSY signal. Events were generated by Monte Carlo simulations according to the cross-section calculation of Ref. [1] and using the EHLQ I structure function parametrizations [14] to describe the initial parton fluxes. The convention of Ref. [14] has been adopted for the running strong coupling constant $\alpha_{S}$ with $\Lambda=200 \mathrm{MeV}$. This generation does not include contributions from unknown higher order terms in $\alpha_{s}$. The cross-section values are typically $30 \%$ lower than those generated according to Ref. [15]. The initial SUSY particle pairs have been simulated with a transverse momentum distribution as given in Ref. [16].

Five mass degenerate $\widetilde{\mathrm{q}}$ flavours were assumed, as well as equal masses for the two q states associated with each left - and right - handed q. Gluinos were fragmented using the prescription of Ref. [17]. Branching ratios of $100 \%$ for $\widetilde{\mathrm{g}} \rightarrow \mathrm{q} \overline{\mathrm{q}} \tilde{\gamma}$ and $\widetilde{\mathrm{q}} \rightarrow \mathrm{q} \widetilde{\mathrm{g}}$ (if $\mathrm{m} \widetilde{\mathrm{q}}>\mathrm{m} \widetilde{\mathrm{g}}$ ) or $\widetilde{\mathrm{q}} \rightarrow \mathrm{q} \boldsymbol{\gamma}$ (if $\mathrm{m} \widetilde{\mathrm{q}}<\mathrm{m} \widetilde{\mathrm{g}}$ ) with isotropic decays were used in the simulation. The $\boldsymbol{\gamma}$ was assumed to be the LSP and nearly massless. The results of the simulations depend on the SUSY model assumptions as discussed in Sect. 5.

The final state $q$ and $g$ were hadronized following the scheme of Ref. [18], optimized to describe jet fragmentation for ordinary QCD 2-jet events. The last step in the simulation consisted of a detailed simulation of the calorimeter response to all final state particles based on extensive test beam studies [10].

The Monte Carlo simulations have been performed for 32 different points in the $(m \tilde{q}, m \tilde{g})$ plane. The results are given in Table 1 for three representative choices of $m \tilde{q}$ and $m \tilde{g}$ which characterize the cases of about equal masses $(m \tilde{q}=99 \mathrm{GeV}, m \tilde{g}=100 \mathrm{GeV})$, of a heavy $\widetilde{q}(m \tilde{q}=200 \mathrm{GeV}, \mathrm{m} \tilde{g}=80 \mathrm{GeV})$, and of a heavy $\widetilde{g}\left(m \tilde{q}=70 \mathrm{GeV}, \mathrm{m} \tilde{\mathrm{g}}=10^{5} \mathrm{GeV}\right)$. The very large $\mathrm{m} \tilde{\mathrm{g}}$ considered in the latter choice is necessary because the $\widetilde{\mathrm{q}} \overline{\mathrm{q}}$ production cross-section [1] depends on $\mathrm{m} \tilde{\mathrm{g}}$ even for $\mathrm{m} \tilde{\mathrm{g}} \gg \mathrm{m} \tilde{\mathrm{q}}$. Listed in Table 1 are the generated 
cross-sections and the accepted fraction $\varepsilon$ of the simulated events after applying all kinematical and topological selections of the same analysis as described in the previous Section. The acceptance $\varepsilon$ is low $(\leq 0.1 \%)$ for light $\widetilde{\mathrm{q}}$ and $\widetilde{\mathrm{g}}$ masses $(<50 \mathrm{GeV})$, reaching values of typically $5 \%$ for the largest masses accessible in the present search. In the following, the model dependence on both the QCD parameters and the instrumental simulation will be discussed.

\subsection{Model dependence on QCD parameters}

Several parameters of the QCD calculations ( $\alpha_{\mathrm{S}}$ and structure functions, $\mathrm{Q}^{2}$-scale, $\mathrm{p}_{\mathrm{T}}$ of the SUSY pair) have been varied in order to estimate the influence of their systematic uncertainties on the expected cross-sections. The effects of these variations are also listed in Table 1 in the form of ranges spanning the observed relative changes of the accepted crosssections with respect to the initial simulation.

The simulations have been repeated with a large variety of $\alpha_{\mathbb{S}}$ values and different structure function parametrizations in order to evaluate the cross-section uncertainty due to the limited knowledge of the incoming parton fluxes. The different sets of parametrizations proposed by Refs. $[14,19-21]$ give results within a range of about $\pm 20 \%$ with respect to the initial cross-section computation. These uncertainties also include variations in the QCD parameter $\Lambda$ which has been set to values between 100 and $400 \mathrm{MeV}$ consistently with the ones needed in the parametrizations of Refs. [14, 19-21].

The initial generation assumed $Q^{2}=\hat{s}$ which is the largest possible $Q^{2}$-scale in the process, where $\sqrt{ } \hat{s}$ is the SUSY pair centre-of-mass energy. Other $Q^{2}$ choices therefore increase the expected cross-sections. The lowest $Q^{2}$-scale considered in Table 1 corresponds to the choice of Ref. [15].

The $\mathrm{p}_{\mathrm{T}}$ of the generated SUSY particle pair has been generated according to the relatively soft distribution computed by Ref. [16] for $\mathrm{W}$ production (mean $\mathrm{p}_{\mathrm{T}} \sim 10 \mathrm{GeV}$ ). Two other models have been used to investigate further the importance of this assumption. SUSY particle pairs were generated on one hand with a harder $\mathrm{p}_{\mathrm{T}}$ distribution derived from the measured distribution for UA2 two-jet data with similar $\sqrt{\hat{s}}$ values (mean $\mathrm{p}_{\mathrm{T}} \sim 20 \mathrm{GeV}$ ). On the other hand the extreme case of zero $\mathrm{p}_{\mathrm{T}}$ for the SUSY particle pair system was also considered. As expected, harder $\mathrm{p}_{\mathrm{T}}$ spectra would in general increase the observable crosssections given the kinematical selections applied in the present analysis. 


\subsection{Model dependence on the instrumental simulation}

The results of the simulation depend also on the modelling of the detector response to the final state $\mathrm{q}$ and $\mathrm{g}$ in the environment of $\overline{\mathrm{p}}$ collisions. The following effects have been investigated (see Table 1) : fragmentation model and jet energy scale, calorimeter response simulation, and influence of the underlying event (contribution from the partons which are not part of the hard scattering process producing the SUSY particle pair).

The uncertainties of the fragmentation scheme and of the calorimeter response simulation are correlated. Of particular importance is the response to low energy fragments due to the non-linearities inherent in hadron calorimetry at energies $\mathrm{E} \leq 2 \mathrm{GeV}$ [10]. The error estimate (Table 1) has been obtained by simultaneously varying the parameters of the adopted fragmentation scheme [18] within limits compatible with jet fragmentation data from $\mathrm{e}^{+} \mathrm{e}^{-}$and $\overline{\mathrm{p}} \mathrm{p}$ collider experiments, by varying the calorimeter response model, and by allowing the absolute energy calibration to change by $\pm 2 \%[10]$.

Finally, the contribution of the underlying event has been investigated by superimposing a variable number of real, unbiased $\vec{p} p$ events onto the Monte Carlo events. The initial simulation was made with no underlying event. Adding the energies of up to 3 unbiased $\bar{p}$ p events did not affect significantly the observed cross-sections due to the selective kinematical criteria. The effect enhances slightly the observable cross-sections but has been conservatively neglected in the present analysis.

\section{RESULTS AND CONCLUSIONS}

At this stage the experimental data can be confronted with the SUSY Monte Carlo expectation. The cross-section limit of $0.35 \mathrm{pb}(90 \% \mathrm{CL})$ for multi-jet events with large $p_{\mathrm{T}}$ as defined by the kinematical and topological configurations described in Sect. 3 is used to exclude a region in the $(m \tilde{q}, m \tilde{g})$ plane for which a significant SUSY signal would be expected from the Monte Carlo model for the present data sample.

The systematic uncertainties discussed in the previous Section are taken into account by lowering the expected observable cross-sections from the model. The different uncertainties have been combined by a Monte Carlo method which computes a combined probability distribution assuming that all error contributions are independent, and that each value be distributed with equal probability over the given error interval. The combined systematic error used for the $90 \% \mathrm{CL}$ calculations is then defined by the value for which the above integrated probability distribution reaches $10 \%$. The combined systematic errors applied for the three representative $(\mathrm{m} \tilde{\mathrm{q}}, \mathrm{m} \tilde{\mathrm{g}})$ points are also listed in Table 1. 
The expected observable cross-sections, lowered by the combined systematic-errors, are shown in Fig. 4 for the cases of nearly equal $\widetilde{\mathrm{q}}$ and $\widetilde{\mathrm{g}}$ masses as well as for large $\widetilde{\mathrm{q}}$ and large $\widetilde{\mathrm{g}}$ masses. The $90 \% \mathrm{CL}$ lower mass limits can be deduced from Fig. 4 directly from the intersection with the experimental cross-section limit. The excluded ( $90 \% \mathrm{CL}$ ) region in the $(\mathrm{m} \tilde{\mathrm{q}}, \mathrm{m} \tilde{\mathrm{g}})$ plane is displayed in Fig. 5. Adding the systematic uncertainties in quadrature instead of using the above Monte Carlo procedure would decrease the mass limits by about $3 \mathrm{GeV}$. Masses below about $50 \mathrm{GeV}$ are not excluded by the present analysis $(\varepsilon<0.1 \%)$.

The mass limits depend on the assumptions on $m \tilde{\gamma}$ and the BR for $\widetilde{q}$ and $\tilde{\mathrm{g}}$ decays in the SUSY model. The dependence on $m \tilde{\gamma}$ has been investigated with Monte Carlo studies. The results of Figs. 4 and 5 have been found to be insensitive to $m \tilde{\gamma}$ for $m \tilde{\gamma}<20 \mathrm{GeV}$. At larger $\mathrm{m} \tilde{\gamma}$ a loss of observable cross-section is caused by the softer $\phi_{\mathrm{T}}$ distributions compared to $m \tilde{\gamma}=0$. This loss is typically $10 \%$ for $m \tilde{\gamma}=30 \mathrm{GeV}$. However for such a heavy $\tilde{\gamma}$ more complex SUSY models $[2,22]$ would have to be considered. Models with an unstable $\tilde{\gamma}, \tilde{\gamma} \rightarrow \gamma \tilde{x}$ with $\tilde{x}$ being the LSP [22], are not considered in the present analysis. In this case the $\phi_{\mathrm{T}}$ spectra are expected to be softer, resulting in lower mass limits. An analysis with less restrictive kinematical criteria which included the additional signature of two photons has been reported previously by UA2 [5].

It has been pointed out $[2,22]$ that heavy $\widetilde{q}$ and $\widetilde{g}$ may not only decay directly to the LSP as assumed in the SUSY model of Sect. 4.1 but also by cascade decays via charginos and neutralinos. In this case the $p_{\mathrm{T}}$ carried by the LSP is again smaller than for the direct decays. As a consequence lower mass limits would be obtained ignoring conservatively any contributions to the observable cross-section from the cascade decays. This degradation has been studied in Ref. [2] for various model parameters. The observable cross-section curves as a function of the SUSY particle masses given in Fig. 4 can be used to estimate the effects on the mass limits in case of reduced BR for the direct decays. Modified limits can be obtained by rescaling the curves by the corresponding $B R$ value.

The previous $\widetilde{\mathrm{q}}$ and $\tilde{\mathrm{g}}$ mass limits at $90 \% \mathrm{CL}$ from UA1 [6] at $\sqrt{\mathrm{s}}_{\mathrm{s}}=630 \mathrm{GeV}$ and from $\mathrm{CDF}[7]$ at $\sqrt{s}_{\mathrm{s}}=1.8 \mathrm{TeV}$ are also indicated in Fig. 5. The present results improve and complement these measurements by excluding new regions in the $(\mathrm{m} \tilde{\mathrm{q}}, \mathrm{m} \tilde{\mathrm{g}})$ plane. The results of Ref. [7] have been obtained with a small data sample only. As the $\widetilde{\mathrm{q}}$ and $\widetilde{\mathrm{g}}$ production cross-sections increase strongly with $\sqrt{s}$, it is expected that higher bounds on the $\widetilde{\mathrm{q}}$ and $\widetilde{\mathrm{g}}$ masses will be reached for data samples comparable to the present experiment.

In conclusion a search for multi-jet events with large $p_{\mathrm{T}}$ has resulted in no event with $p_{\mathrm{T}}>40 \mathrm{GeV}$ for kinematical and topological configurations which would retain a significant sample of SUSY events with $\widetilde{\mathrm{q}}$ and $\widetilde{\mathrm{g}}$ masses above the previous lower limits. Under the assumption of a minimal SUSY model with $m \widetilde{\gamma}<20 \mathrm{GeV}$ and no cascade decays [2] of the $\widetilde{\mathrm{q}}$ and $\widetilde{\mathrm{g}}$ to the $\gamma$ (LSP), the following $90 \%$ CL lower mass limits are obtained : 
$\mathrm{m}_{\tilde{q}}>74 \mathrm{GeV}$ independent of $\mathrm{m} \tilde{\mathrm{g}}, \mathrm{m} \tilde{\mathrm{g}}>79 \mathrm{GeV}$ independent of $\mathrm{m} \tilde{q}$, and $\mathrm{m}>106 \mathrm{GeV}$ for $m_{\tilde{q}}=m \tilde{g}=m$. Masses below about $50 \mathrm{GeV}$ are not excluded by this analysis.

\section{ACKNOWLEDGEMENTS}

We gratefully acknowledge P. Darriulat for his contributions and guidance during the design and construction phases of the UA2 upgrade project.

The technical staff of the institutes collaborating in UA2 have contributed substantially to the construction and operation of the experiment. We deeply thank them for their continuous support.

The experiment would not have been possible without the very successful operation of the improved CERN $\vec{p}$ Collider whose staff and coordinators we sincerely thank for their collective effort.

Financial support is acknowledged from the Schweizerischen Nationalfonds zur Förderung der Wissenschaftlichen Forschung to the Bern group, from the UK Science and Engineering Research Council to the Cambridge group, from the Bundesministerium für Forschung und Technologie to the Heidelberg group, from the Institut National de Physique Nucléaire et de Physique des Particules to the Orsay group, from the Istituto Nazionale di Fisica Nucleare to the Milano, Pavia, Perugia and Pisa groups and from the Institut de Recherche Fondamentale (CEA) to the Saclay group. 
Table 1

SUSY Model Calculations

\begin{tabular}{|c|c|c|c|c|c|c|}
\hline & \multicolumn{2}{|c|}{$\begin{array}{l}\mathrm{m}_{\tilde{\mathrm{q}}}=99 \mathrm{GeV} \\
\mathrm{m} \tilde{\mathrm{g}}=100 \mathrm{GeV}\end{array}$} & \multicolumn{2}{|c|}{$\begin{array}{l}\mathrm{m} \tilde{\mathrm{q}}=200 \mathrm{GeV} \\
\mathrm{m} \tilde{\mathrm{g}}=80 \mathrm{GeV}\end{array}$} & \multicolumn{2}{|c|}{$\begin{array}{l}\mathrm{m} \tilde{\mathrm{q}}=70 \mathrm{GeV} \\
\mathrm{m} \tilde{\mathrm{g}}=10^{5} \mathrm{GeV}\end{array}$} \\
\hline $\begin{array}{ll}\text { cross-sections : } & \\
\text { produced } & (\mathrm{pb}) \\
\text { observed } & (\mathrm{pb}) \\
\varepsilon & (\%)\end{array}$ & $\begin{array}{c}9.4 \\
0.60 \\
6.3\end{array}$ & & $\begin{array}{c}14.4 \\
0.45 \\
3.1\end{array}$ & & $\begin{array}{r}14 . \\
0.46 \\
3.2\end{array}$ & \\
\hline $\begin{array}{l}\text { systematic errors }(\%): \\
\alpha_{S}+\text { structure functions } \\
\mathrm{Q}^{2} \text {-scale } \\
\mathrm{p}_{\mathrm{T}} \text { SUSY pair } \\
\text { jet energy scale } \\
\text { (including fragmentation) }\end{array}$ & $\begin{array}{r}\text { low } \\
-11 \\
0 \\
-9 \\
-11\end{array}$ & $\begin{array}{c}\text { high } \\
22 \\
16 \\
4 \\
16\end{array}$ & $\begin{array}{c}\text { low } \\
-18 \\
0 \\
-5 \\
-23\end{array}$ & $\begin{array}{c}\text { high } \\
20 \\
14 \\
0 \\
43\end{array}$ & $\begin{array}{r}\text { low } \\
-16 \\
0 \\
0 \\
-16\end{array}$ & $\begin{array}{c}\text { high } \\
20 \\
16 \\
16 \\
26\end{array}$ \\
\hline $\begin{array}{l}\text { combined systematic } \\
\text { error for } 90 \% \mathrm{CL}\end{array}$ & & & & & & \\
\hline
\end{tabular}




\section{References}

[1] S. Dawson, E. Eichten and C. Quigg, Phys. Rev. D31 (1985) 1581.

[2] H. Baer et al., Phys. Lett. B161 (1985) 175;

H. Baer et al., Phys. Rev. Lett. 63 (1989) 352.

[3] E. Jones et al., IEEE Trans. Nucl. Sci. NS-30 (4) (1988) 2778;

E.Jones, Proc. 6th Topical Workshop on Proton-Antiproton Collider Physics, Aachen, 1986, eds. K. Eggert et al., (World Scientific, Singapore, 1987), p. 691.

[4] UA1 Collaboration, G. Arnison et al., Phys. Lett. B139 (1984) 115.

[5] UA2 Collaboration, R. Ansari et al., Phys. Lett. B195 (1987) 613.

[6] UA1 Collaboration, C. Albajar et al., Phys. Lett. B198 (1987) 261.

[7] CDF Collaboration, F. Abe et al., Phys. Rev. Lett. 62 (1989) 1825.

[8] UA2 Collaboration, T. Akesson et al., to be submitted to Phys. Lett. B.

[9] UA2 Collaboration, Proposal to Improve the Performance of the UA2 Detector, CERN/SPSC 84-30, 84-95, and 85-3 (1984 and 1985, unpublished); UA2 Collaboration, C.N. Booth, Proc. 6th Topical Workshop on ProtonAntiproton Collider Physics, Aachen, 1986, eds. K. Eggert et al. (World Scientific, Singapore, 1987), p. 381.

[10] F. Alberio et al., The Electron, Jet, and Missing Transverse Energy Calorimetry of the Upgraded UA2 Experiment at the CERN $\overrightarrow{p p}$ Collider, in preparation for Nucl. Instr. Meth. .

[11] G. Blaylock et al., Proc. of the Intemational Conference on the Impact of Digital Microelectronics and Microprocessors on Particle Physics 1988, eds. M. Budinich et al. (World Scientific, Singapore, 1988), p. 247 and 254.

[12] A. Beer et al., Nucl. Instr. Meth. 224 (1984) 360. 
[13] P. Moreira and G. Polesello, CERN-EP/89-110, to appear in Nucl. Instr. Meth. .

[14] E. Eichten et al., Rev. Mod. Phys. 56 (1984) 579 and 58 (1986) 1065 E.

[15] F. Paige and S. Protopopescu, ISAJET programme, BNL 38034 (1986).

[16] G. Altarelli et al., Nucl. Phys. B246 (1984) 12.

[17] A. DeRujula and R. Petronzio, Nucl. Phys. B261 (1985) 1587.

[18] R.D. Field and R.P. Feynman, Nucl. Phys. B136 (1978) 1.

[19] D.W. Duke and J.F. Owens, Phys. Rev. D39 (1984) 49.

[20] A.D. Martin et al., Phys. Rev. D37 (1988) 1161 and Phys. Lett. B206 (1988) 327.

[21] M. Diemoz et al., Z. Phys. C39 (1988) 21.

[22] A selected sample of Refs. include :

P. Fayet, Unification of the fundamental particle interactions, eds. S. Ferrara et al., (Plenum, NY, 1980) 587;

H.P. Nilles, Phys. Rep. 110 (1984) 1;

J. Ellis et al., Nucl. Phys. B238 (1984) 453;

H.E. Haber and G.L. Kane, Phys. Rep. 117 (1985) 75;

G. Gamberini, Z. Phys. C30 (1986) 605;

R. Barbieri et al., Nucl. Phys. B301 (1988) 15. 


\section{Figure Captions}

Fig. 1 A view of the upgraded UA2 detector in a plane containing the beam line.

Fig. $2 \quad p_{\mathrm{T}}^{2}$-distributions for two representative $\mathrm{E}_{\mathrm{T}}$ intervals :
(a) $30<\mathrm{E}_{\mathrm{T}}<40 \mathrm{GeV}$, (b) $110<\mathrm{E}_{\mathrm{T}}<120 \mathrm{GeV}$.

Fig. $3 \quad \mathbf{p}_{\mathrm{T}}^{2}$-distributions for the present multi-jet data with large $\boldsymbol{p}_{\mathrm{T}}$. The data are shown before the $p_{\mathrm{T}}$ isolation and electron rejection requirements (see text) and after all selections applied (shaded histogramme).

Fig. 4 Expected observable cross-sections as a function of the SUSY particle mass after all selection criteria and including the systematic uncertainties needed to deduce $90 \%$ CL mass limits. The present experimental cross-section limit $(90 \% \mathrm{CL})$ is also indicated.
(a) Nearly equal $\tilde{\mathrm{q}}$ and $\tilde{\mathrm{g}}$ masses $(\mathrm{m} \tilde{\mathrm{q}}=\mathrm{m} \tilde{\mathrm{g}}-1 \mathrm{GeV})$,
(b) heavy $\tilde{\mathrm{q}}$ case $\left(\mathrm{m}_{\tilde{\mathrm{q}}}=200 \mathrm{GeV}\right)$, and (c) heavy $\widetilde{\mathrm{g}}$ case $\left(\mathrm{m} \tilde{\mathrm{g}}=10^{5} \mathrm{GeV}\right)$.

Fig. 5 Mass region ( $\mathrm{m} \tilde{\mathrm{q}}, \mathrm{m} \tilde{\mathrm{g}})$ excluded by the present experiment (shaded area) with the SUSY model described in the text. The dashed curves indicate the previous mass limits from UA1 [6] and the dashed-dotted curve the one from CDF [7]. All limits are for $90 \% \mathrm{CL}$. 


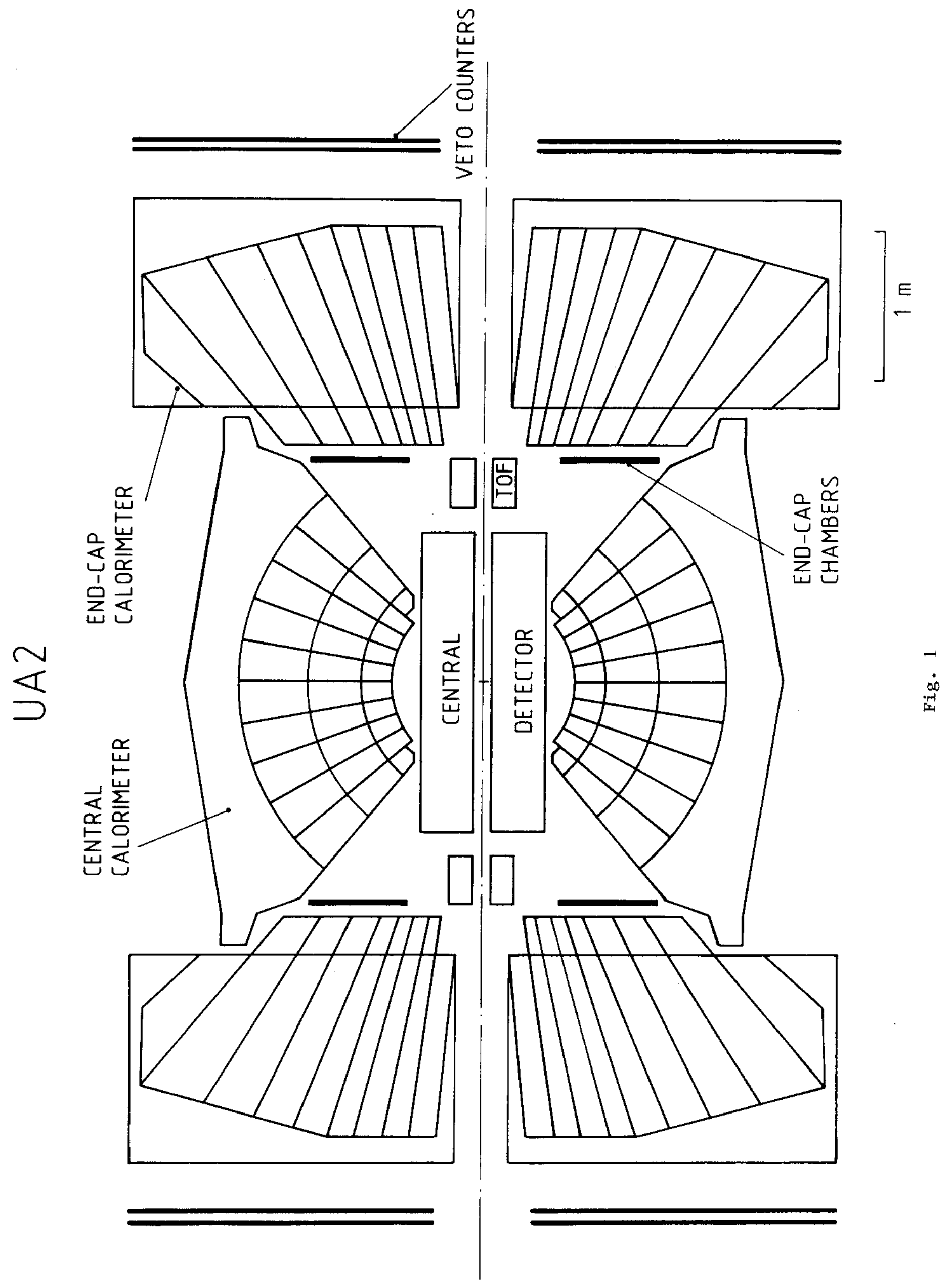



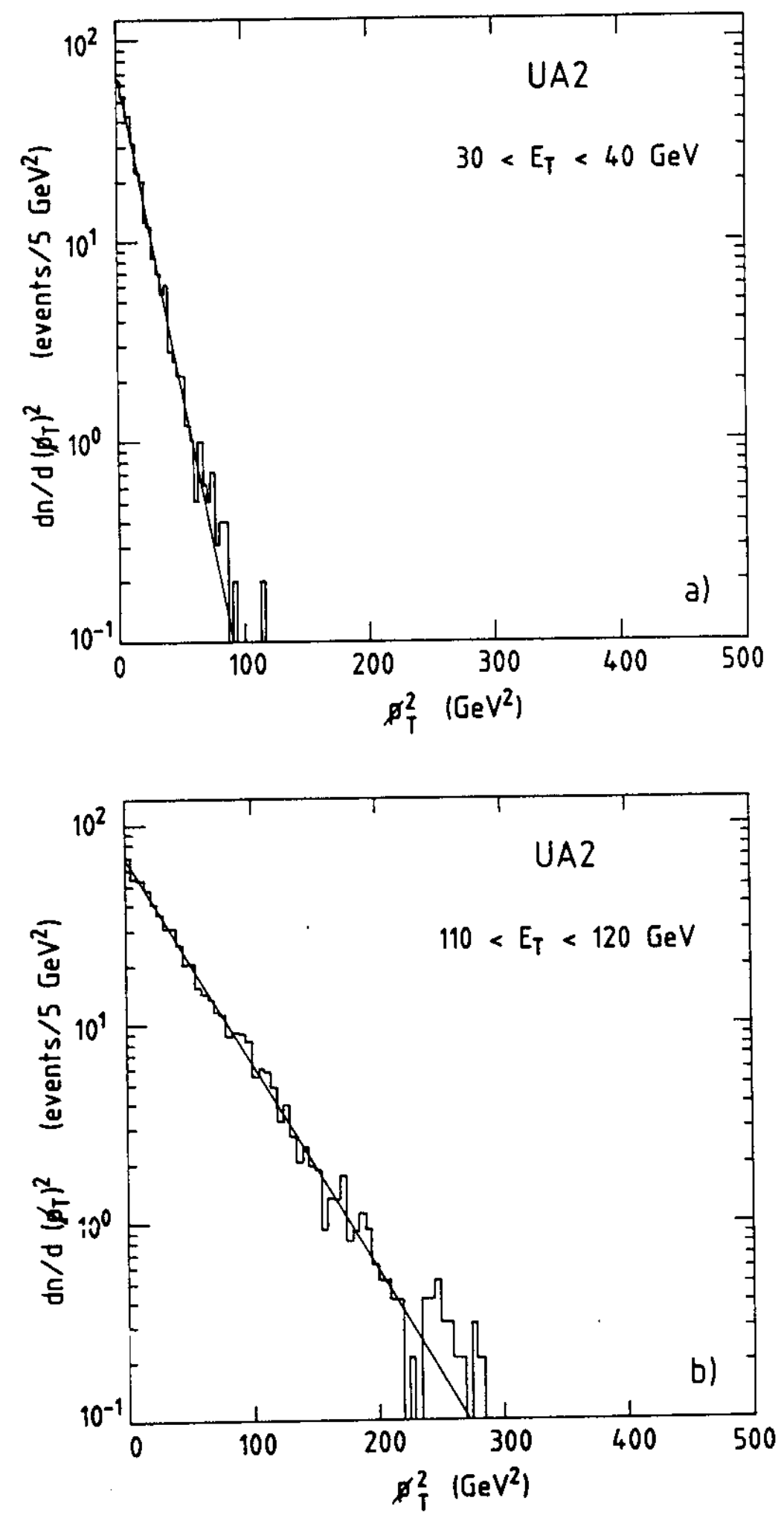

Fig. 2 


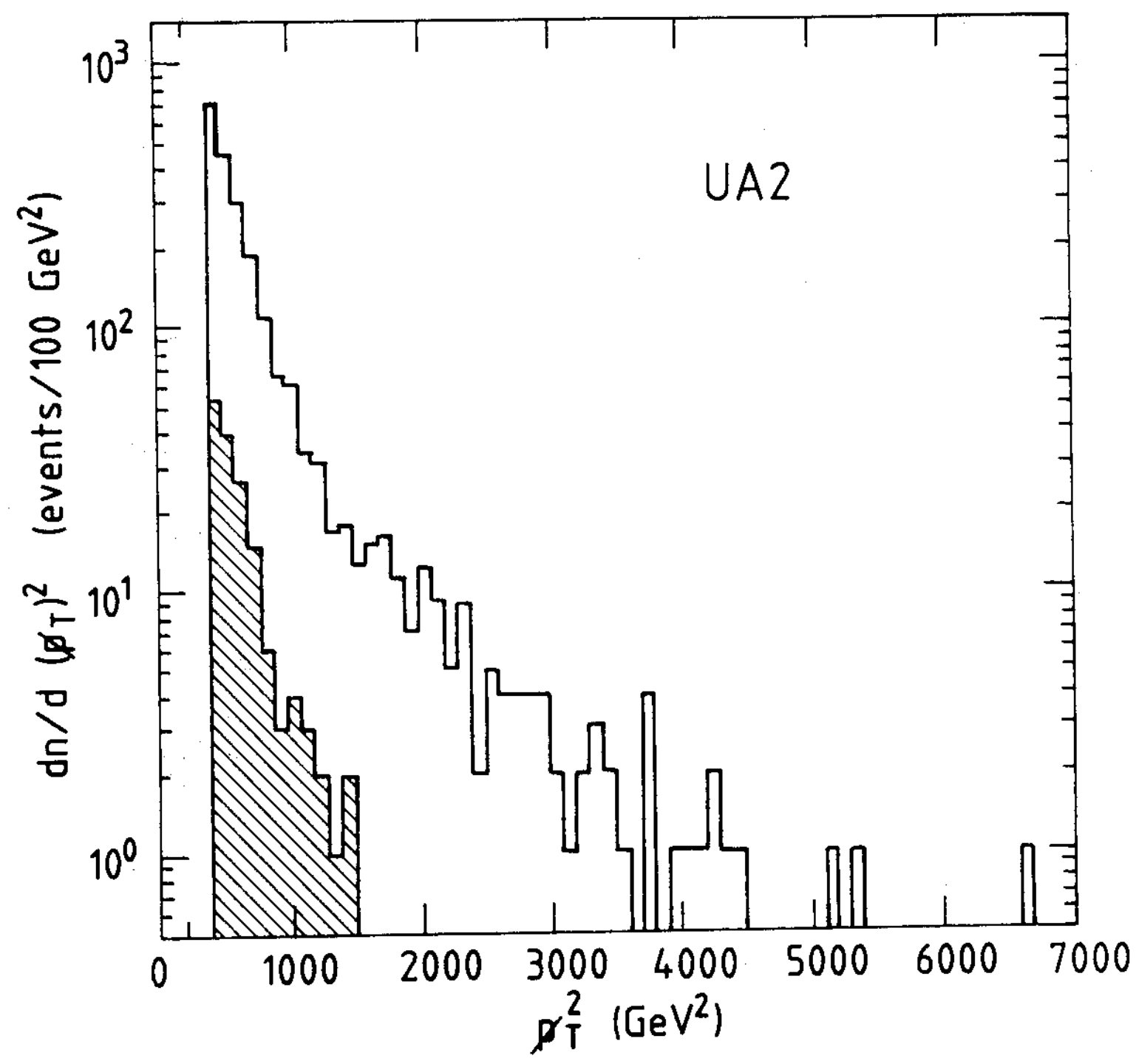

Fig. 3 


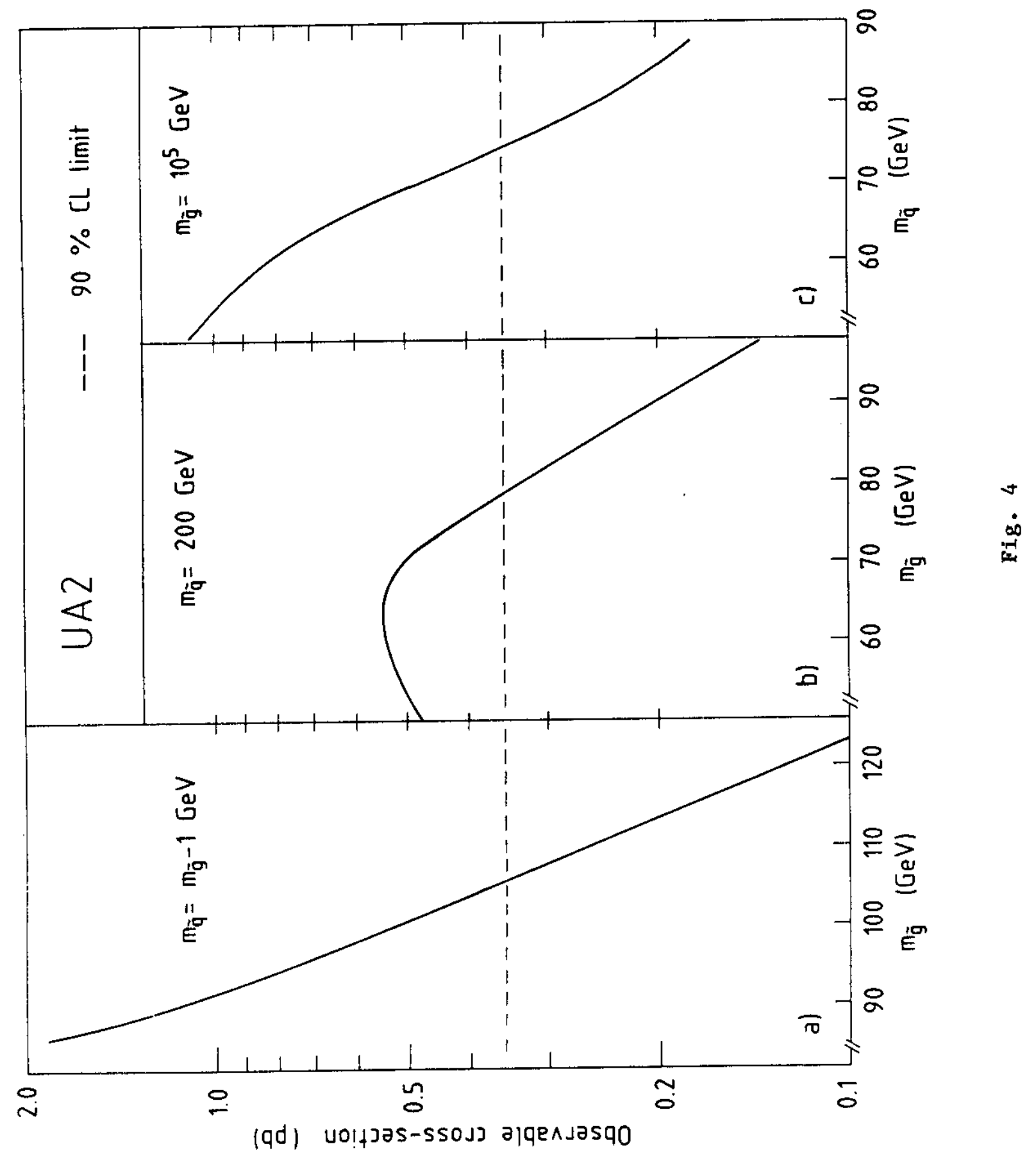




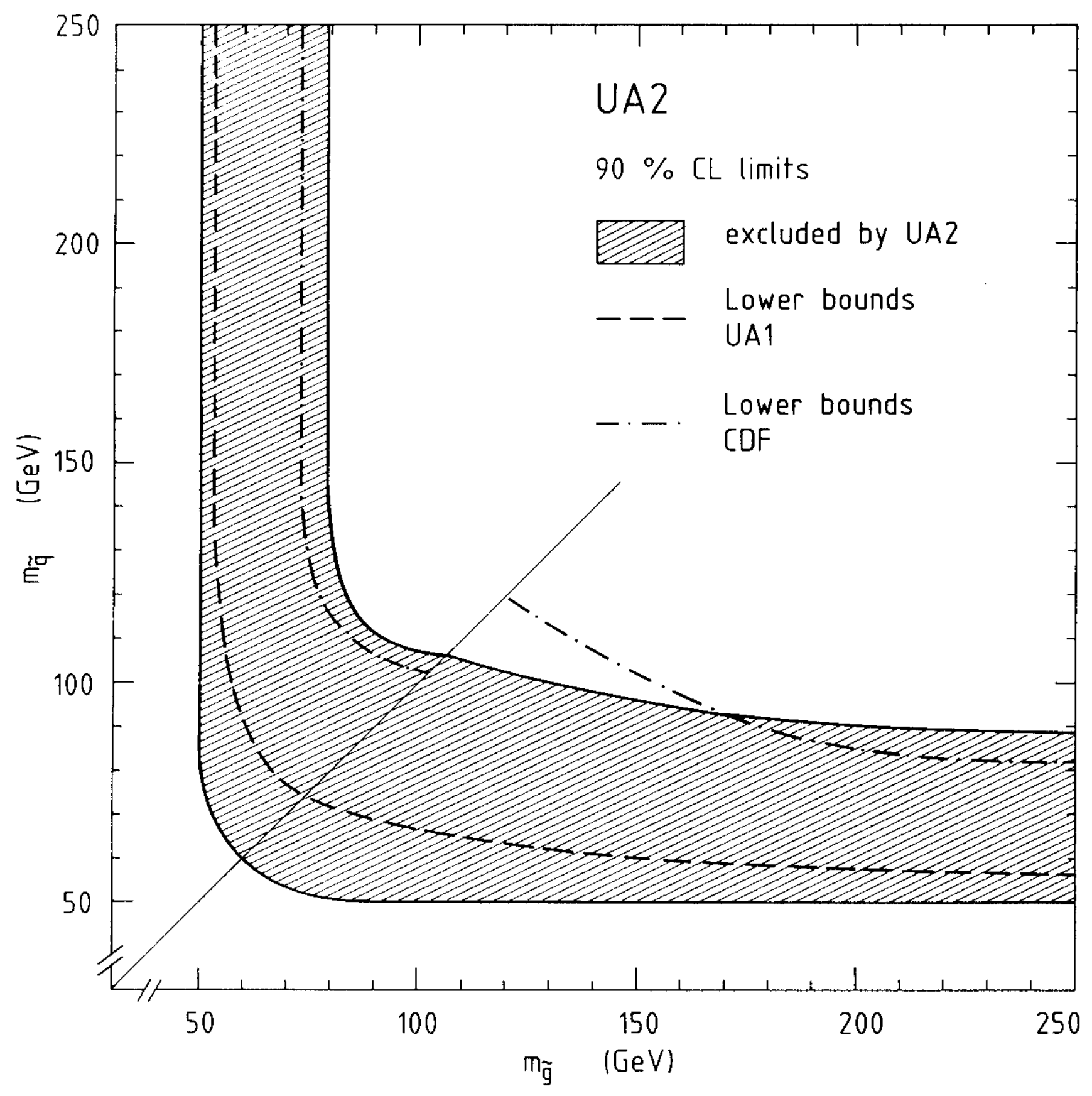

Fig. 5 